\title{
Maternal Dietary Exposure of Zinc Oxide Nanoparticles (ZnONPs) and its Effect in Guppies (Poecilia reticulata)
}

\author{
Subburaman Chandramohan, Krishnan Sundar, Azhaguchamy Muthukumaran
}

\begin{abstract}
Zinc deficiency is one of the major malnutritional problems worldwide specifically in developing and developed countries. The zinc uptake takes place in human body in ionic form and metabolized naturally and converted into nano form. In the present study zinc oxide nanoparticles (ZnONPs) were synthesized by starch. The synthesized different ZnONPs were characterized by Fourier Transform Infra-Red spectroscopy (FTIR) and Scanning Electron Microscopy (SEM) coupled with Energy Dispersive $X$ ray analysis $(E D X)$. FTIR analysis revealed Zinc peak at $405.05 \mathrm{~cm}^{-1}$.The average size of the synthesized ZnONPs was in the range from 200-300 $\mathrm{nm}$ with flower like shape. Furthermore, toxicity and level of nanoparticle accumulation were evaluated in young and adult guppies (Poecilia reticulata) using different concentration of ZnONPs. To explore the toxicological effect, the different concentration of nanoparticles administered in young and adult guppies. There was a dose dependent toxicological effects were noted in the guppies.
\end{abstract}

Keywords- Zinc oxide nanoparticles (ZnONPs), Guppies (Poecilia reticulata), toxicological effect, accumulation

\section{INTRODUCTION}

$\mathrm{N}_{\mathrm{a}}$ anotechnology is a major field involved in designing and fabrication of materials for biomedical applications. The main reason of using nanoparticles is that they have larger surface area which increases the cellular uptake of nanoparticles. Some of the applications of nanotechnology includes electronics such as photo detectors, are the sensors of light that converts light to current and may be used as safety devices in homes like smoke detectors. Sensors, that detects physical properties and its recording and for medical diagnostics purposes. Solar cells are used as photo detectors that convert sunlight to electric energy.

The purpose of using Zinc Oxide nanoparticles have been found to have superior UV blocking properties compared to other particles. It is mainly used to block UV rays that provide larger UVA protection. UV-A is a long wave which

Revised Manuscript Received on December 16, 2019.

* Correspondence Author

Subburaman Chandramohan, Department of Biotechnology, Kalasalingam Academy of Research and Higher Education, Krishnankoil, Tamil Nadu 626126, India. Email: chandramohan@klu.ac.in

Krishnan Sundar, Department of Biotechnology, Kalasalingam Academy of Research and Higher Education, Krishnankoil, Tamil Nadu 626126, India. Email: sundarkr@klu.ac.in

Azhaguchamy Muthukumaran*, Department of Biotechnology, Kalasalingam Academy of Research and Higher Education, Krishnankoil, Tamil Nadu 626126, India. Email: a.muthukumaran@klu.ac.in penetrates deep on the surface and so, they often react quickly. The unique properties of zinc oxide nanoparticles have been found to be is that have wide band gap (energy difference between bands) $3.3 \mathrm{eV}$ with low toxicity, low processing cost for II-IV semiconductors. It also has high refractive index (ratio of velocity of light in vacuum) around 1.9, suitable for reduce scattering [1] [2] [3]. It has high exciton binding energy of $60 \mathrm{meV}$ at room temperature that ensures efficient luminescent characteristics due to this it is useful for the development of many optoelectronic devices, specifically in photo luminescent spectrum of $\mathrm{ZnO}$ nanoparticles with very sharp peaks are observed [4].

There are some of the techniques used to characterize the synthesized nanoparticles. The synthesis of nanoparticles involves three methods namely, biological, chemical, physical and mechanical methods. In this process, biological method is preferred because, we can synthesis nanoparticles using plants, microbes, fungi and proteins by this method. Here we propose a method for zinc oxide nanoparticles (ZnONPs) by using starch.

In animals, females are more vulnerable to nanoparticles toxicity and it may affect reproductivity and fetal development. Normal female reproduction and fetal development are essential for the perpetuation of the species. So, toxicity testing is an important process and done through safety assessment that refers to determine the degree to which a substance can damage an organism. Using nanoparticles, sequestration can be done in animals that have reproductive impacts [5]. The female reproductive system is different from other system. In a lifespan of a woman, only 400 follicles mature and ovulate. Third, the disturbance of female reproduction inevitably leads to abnormal fetal development. Various environmental chemicals were reported to have deleterious effects on the female reproductive system and embryonic development. The toxicity of nanoparticles to female reproductive and developmental health has been studied in various models. In this research work, we focus on nanotoxicity studies that use guppies as models [6]. And there is also a close similarity between the genome of guppies and humans. There are various advantages of using guppies as an animal model. Other experimental animals are expensive, the study cycle is rather long, the study throughput is low and several animal protection organizations are active around the world. Therefore, the guppies have become the model of choice for molecular mechanism studies in nanotoxicity sequestration and one of the most valued models in developmental biology. The choice of animal plays an important role on sequestration because the choice of animal should be simple with widely

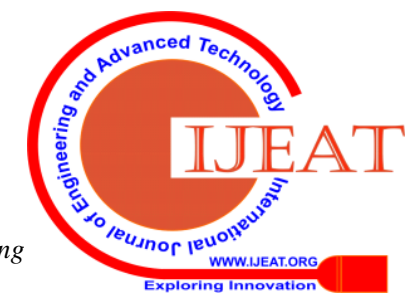


accepted ethical principles and cost of each experiment is expected to be less than others. Hence, guppies are widely preferred due to their cost, availability and maintenance. The guppies are mainly chosen of guppies because, they have reproductivity similar to human and the main purpose is for drug delivery system. The main features of choosing guppies are viviparous i.e., it gives birth to young ones. Guppies, Poecilia reticulata is also known as million fish or rainbow fish. It is a freshwater aquarium fish species and it prefer water temperature around $22.2-26^{\circ} \mathrm{C}$ for reproduction. Guppies can survive both in freshwater as well as saline water. They are used as a model organism in fields of ecology, evolution and behavioral studies. Guppies exhibit sexual dimorphism which makes the males and females distinctly different from each other, while males are more colorful and smaller than female. They also used for controlling mosquito spreading.

\section{MATERIALS AND METHODS}

\section{A. Materials}

In present study, Starch and Zinc acetate were purchased from Hi media, Mumbai, India.

\section{B. Synthesis of Zinc oxide nanoparticles (ZnONPs)}

Soluble starch at different concentrations $(0.3 \%, 0.6 \%, 0.9 \%)$ were mixed with $25 \mathrm{ml}$ of Deionized water. Zinc acetate stock solution of $25 \mathrm{Mm}$ was prepared and mixed with different concentration of starch solutions. The above mixture was autoclaved for $15 \mathrm{~min}$. The obtained reaction mixture was stored at $4{ }^{\circ} \mathrm{C}$ in a refrigerator for further use.

\section{Purification of the ZnONPs}

The synthesized $\mathrm{ZnONPs}$ was purified by centrifugation in $10,000 \mathrm{rpm}$ for $10 \mathrm{mins}$ at $4^{\circ} \mathrm{C}$ in a cooling centrifuge. This process was repeated at least 2-3 times. The supernatant was removed and the pellet was stored for further analysis. The aggregated particles were dispersed by Sonication and the process was repeated at least 2-3 times. Then the pellet was dried to get fine powder.

\section{Characterization of $\mathrm{ZnONPS}$}

The optical characteristics of the synthesized ZnONPs were studied by UV-Visible spectroscopy analysis. ZnONPs (1 ml) nanosuspension was taken for analysis. For analysis Jasco V-650, Germany was used. The synthesized ZnONPs from the starch were analyzed for the presence of functional groups using Fourier Transform Infrared Spectrophotometer at range within from $400 \mathrm{~cm}^{-1}$ to $4000 \mathrm{~cm}^{-1}$ [IR Tracer, Shimadzu, Japan] Kbr pellet method [7]. The size and shape of the ZnO NPs were measured using a Scanning Electron Microscope (EVO-18 Bruker, Germany). Selected areas within the SEM sections were chosen and elemental distribution analysis was done using an Energy Dispersive X-ray spectroscopy (EDX) (Bruker 6130, Germany) microanalysis system coupled with SEM.

\section{E. Experimental design:}

Guppy fish was acclimatized to constant laboratory conditions (14h light: $10 \mathrm{~h}$ dark photo period at $28^{\circ} \mathrm{C}$ ) for at least one week in stock aquaria before experiments were conducted. The feed of the fish was mixed with the ZnONPs at different concentration. Then the fish were fed twice daily blood worms for seven days. The guppies were divided into four groups followed by,
Group 1 - Normal untreated guppy fish young and adult (Control)

Group 2 -Male and female guppies at $0.3 \%$ of $\mathrm{ZnO}$ NPs. Group 3- Male and female guppies at $0.6 \%$ of $\mathrm{ZnO}$ NPs. Group 4 -Male and female guppies at $0.9 \%$ of $\mathrm{ZnO}$ NPs.

\section{F. Exposure of $\mathrm{ZnO}$ nanoparticles to guppies through Feed}

\section{Diet preparation}

Freeze dried worms was fortified with $0.3 \%, 0.6 \%, \& 0.9 \%$ of ZnONPs. The required concentration of each ZnONPs was dissolved into Milli Q water to make $15 \mathrm{ml}$ solution. The prepared solution was poured over $20 \mathrm{~g}$ of dry feed in a Petri dish. It was made sure, that the liquid was evenly distributed in the feed (Fig. 1). The mixture was then freeze-dried overnight. A control diet was made using the same protocol without the addition of $\mathrm{ZnO}$ NPs.

\section{G. Exposure of $\mathrm{ZnO}$ to guppies}

Adult male and female guppies were fed with $\mathrm{ZnO}$ nanoparticles fortified feed of different concentrations $(0.3$, $0.6 \& 0.9 \%$ ) (Fig. 2). The animals were fed twice a day for a week. Another group of guppies including male and female were fed twice a day with the control diet for seven days.

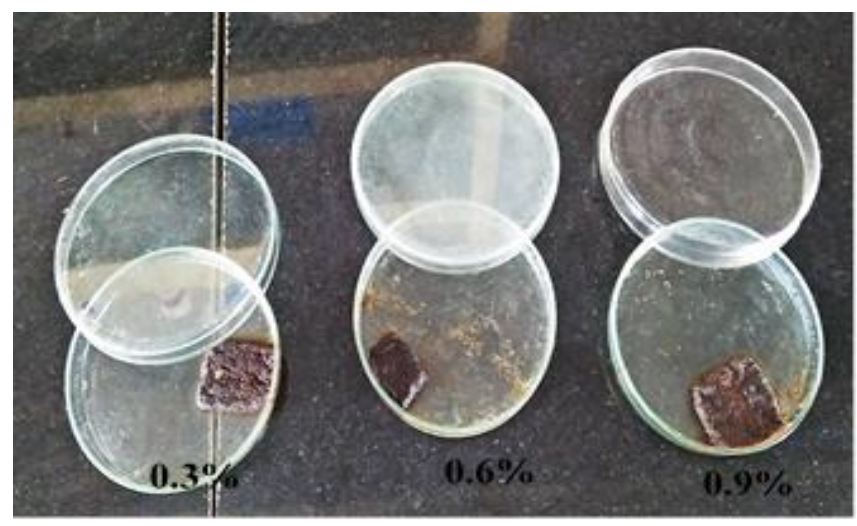

Fig.1. ZnO NP and feed formulation

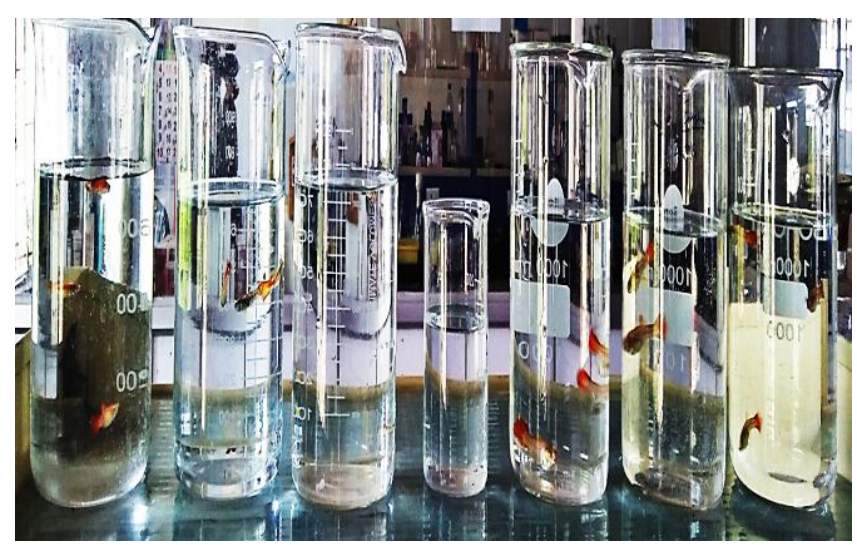

Fig.2. Exposure of $\mathrm{ZnO}$ NPs to guppies

\section{H. Breeding of guppies}

Males and females from individual groups which were fed with ZnONPs diet was paired and allowed to breed for a week. And another group containing male and female guppies fed with the control

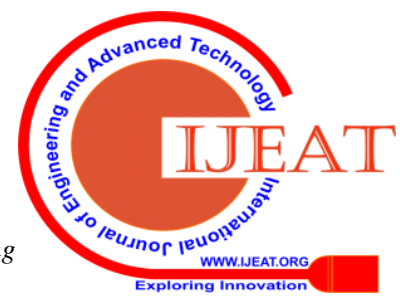


diet were also paired and allowed to breed. During breeding period, the condition of 14:10 light and dark was maintained. The water temperature was maintained at $25^{\circ} \mathrm{C}$.

\section{Toxicity evaluation}

The female guppies which were exposed to $\mathrm{ZnONPs}$ of different concentrations were evaluated for toxicity studies. Various factors such as survival, malformations in hatchings and hatching time were studied and documented.

\section{RESULTS AND DISCUSSION}

\section{A. Characterisation of ZnONPs}

Visible UV-spectroscopy analysis revealed the formation of $\mathrm{ZnO}$ NPs uniformly at the region of $220-300 \mathrm{~nm}$ (Fig. 3)
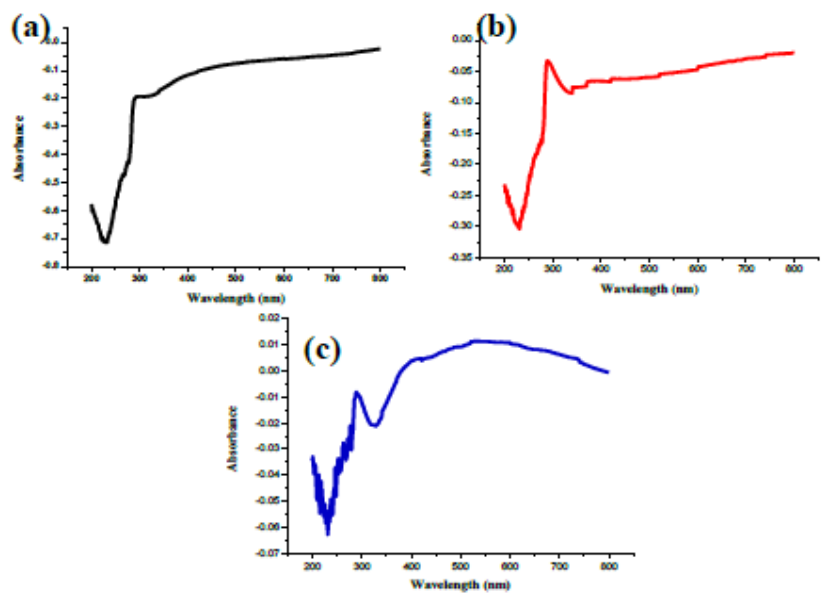

Fig.3. a. UV analysis of ZnONP using $0.3 \%$ starch; b. UV analysis of ZnONP using $0.6 \%$ starch; c. UV analysis of ZnONP using $0.9 \%$ starch.

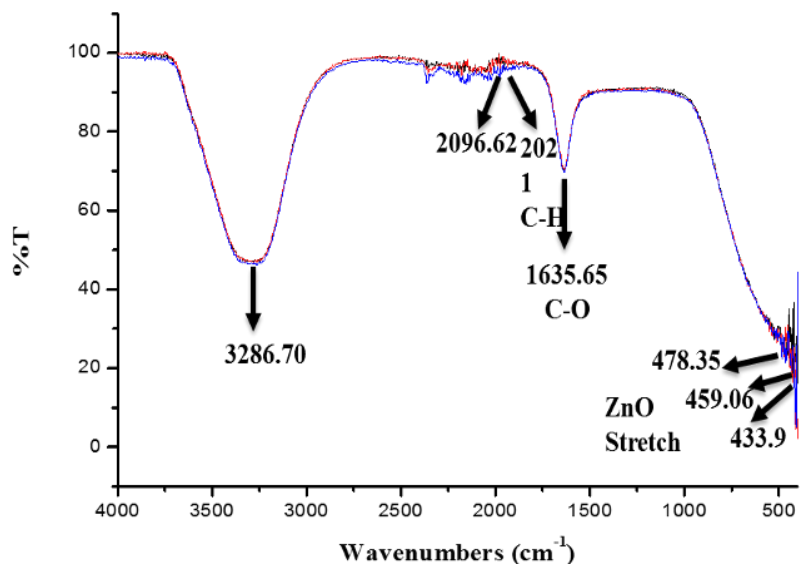

Fig.4 .FTIR of ZnO NPs.
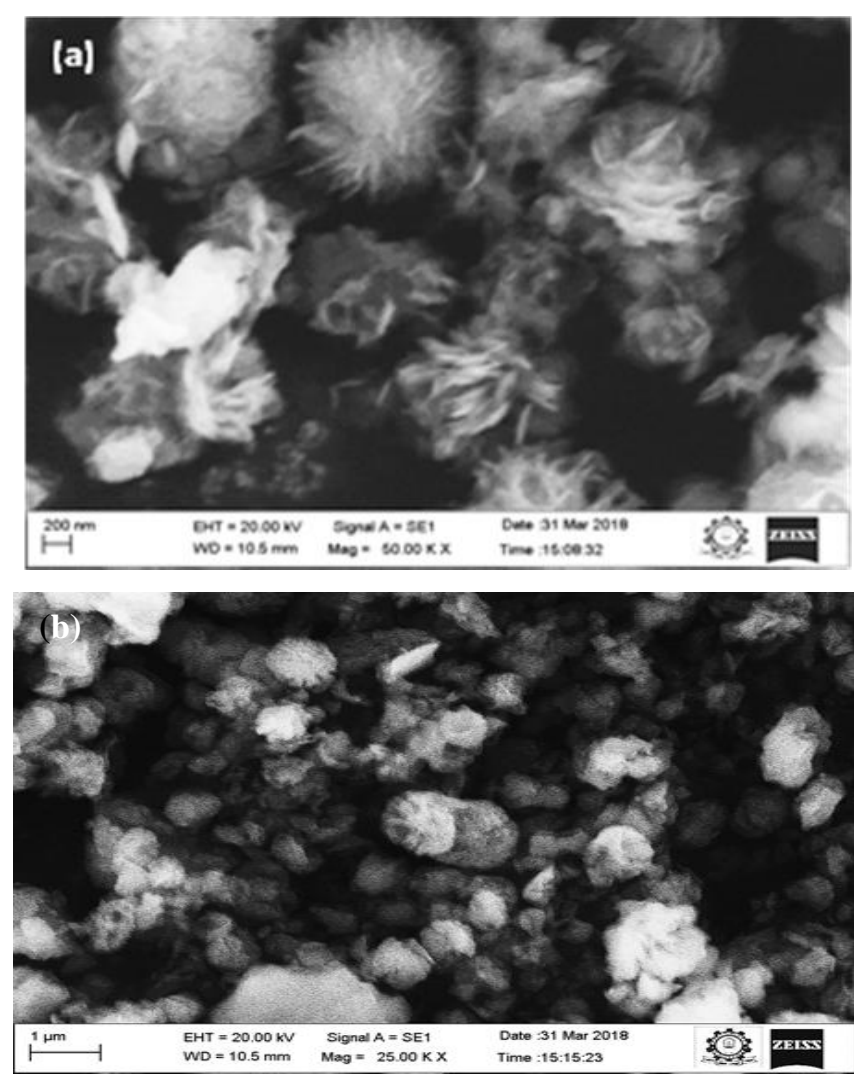

Fig.5. a \& b. SEM analysis of synthesized ZnO NPs.

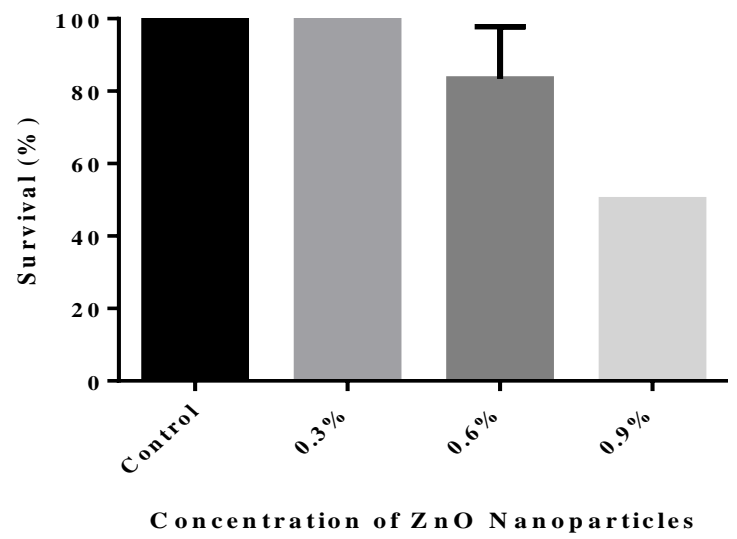

Fig.6. survival rate of new born larvae

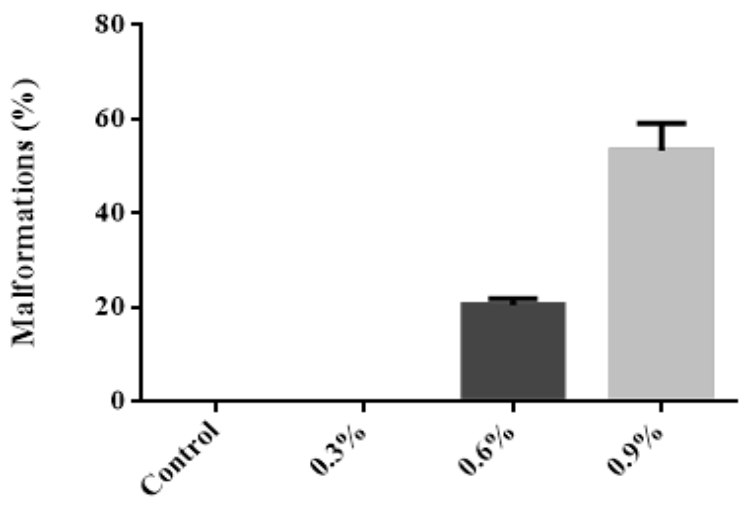

Concentration of $\mathrm{ZnO}$ Nanoparticles

Fig.7. Malformations in the larvae.

Published By:

Blue Eyes Intelligence Engineering \& Sciences Puhlication 
The synthesized ZnO NPs were further characterized by FTIR analysis. Kbr pellet method was used for the FTIR analysis. (Fig. 4) showed the FTIR spectrum of the synthesized $\mathrm{ZnO}$ NP of different concentrations. The various functional groups responsible for the synthesis of $\mathrm{ZnO} \mathrm{NPs}$ such as $\mathrm{C}-\mathrm{H}$ stretch and $\mathrm{C}-\mathrm{O}$ stretch were seen. The region around $435-480 \mathrm{~cm}-1$ denoted the $\mathrm{ZnO}$ finger print region.

The morphology of the synthesized $\mathrm{ZnO}$ NPs were determined Scanning electron microscopy analysis. Results revealed that the synthesized $\mathrm{ZnO}$ NPs were in size ranging from 200-300 $\mathrm{nm}$ and flower like in appearance (Fig. 5)

\section{B. Toxicity evaluation}

The toxicological parameters such as survival of the larvae and malformations were noticed and documented in the nanoparticle exposed guppies (Fig. 6). The survival rate of the $\mathrm{ZnO}$ exposed groups $(0.3,0.6 \& 0.9 \%)$ were studied the survival rate of the larvae decreased in higher concentration $(0.9 \%)$. Almost zero mortality of the new-borns were seen in $0.3 \%$ group and $80 \%$ survival was seen in $0.6 \%$ group. Other malformations such as curved body, irregular heart beat rate and pulmonary edema were also documented (Fig. 7). There were no malformations observed in Control and $0.3 \%$ groups. Abnormalities such as tail malformation were observed in $0.6 \%$ group. Other anomalies like twisted notochord, pulmonary edema and irregular heart beat rate were seen in higher concentration exposed group $(0.9 \%)$.

\section{CONCLUSION}

In the present study $\mathrm{ZnONPs}$ sequestration in gestational period of guppies was studied. ZnONPs was administered through feed to the studied guppies (Adult male and Females). The ZnONPs at higher concentrations $(0.9 \%)$ caused malformations in the offspring. However, zero malformations or very less abnormalities were seen in lower concentration groups $(0.3 \& 0.6 \%)$. Thus, it was evident from the current study lower concentrations $(0.3 \& 0.6 \%)$ can be useful in eliciting various biomedical properties without causing much damage to the system. Still studies on accumulation of $\mathrm{ZnONPs}$ on guppies have to be elaborately made in future.

\section{ACKNOWLEDGMENT}

Authors are grateful to the Kalasalingam Academy of Research and Education for providing funding and research facilities.

\section{REFERENCES}

1. K.R. Raghupathi, R.T. Koodali and A.C. Manna, "Size-dependent bacterial growth inhibition and mechanism of antibacterial activity of zinc oxide nanoparticles", ACS Nano, vol. 27, pp.4020-4028, 2011.

2. L. Zhang, Y. Jiang, Y. Ding, M. Povey and D. York, "Investigation into the antibacterial behaviour of suspensions of $\mathrm{ZnO}$ nanoparticles $(\mathrm{ZnO}$ nanofluids)", Journal of Nanoparticle Research, vol. 9, pp.479-489, 2006.

3. J. Sawai, "Quantitative evaluation of antibacterial activities of metallic oxide powders $(\mathrm{ZnO}, \mathrm{MgO}$ and $\mathrm{CaO})$ by conductimetric assay", $J$ Microbiol Methods, vol. 54, pp. 177-182, 2003.

4. S. Liang, H. Sheng, Y. Liu, Z. Huo, Y. Lu and H. Shen, "ZnO Schottky ultraviolet photodetectors". J Cryst Growth vol. 225, pp. 110-113, 2001.

5. S. Jeyabharathi, K. Kalishwaralal, K. Sundar and A. Muthukumaran, "Synthesis of zinc oxide nanoparticles (ZnONPs) by aqueous extract of Amaranthus caudatus and evaluation of their toxicity and antimicrobial activity", Materials Letters, vol. 209, pp. 295-298, 2017.

6. Jianling Sun, Qiu Zhang, Zhiping Wangand and Bing Yan, "Effects of Nanotoxicity on Female Reproductivity and Fetal Development in Animal Models", International Journal ofMolecular Sciences ISSN 1422-0067, 2013.

7. K. Narimato, 'Infrared and raman spectra of inorganic and coordination compounds. New York: John wiley and sons, pp.296-346. 1997.

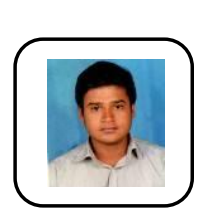

\section{AUTHORS PROFILE}

Mr. S. Chandramohan is currently pursuing $\mathrm{Ph} . \mathrm{D}$ in Biotechnology at Department of Biotechnology, Kalasalingam Academy of Research and Education. His research interests in Nanobiotechnology, Drug delivery and Safety assessment of nanoparticles.

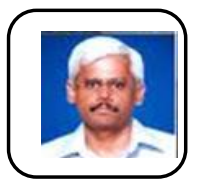

Dr. K. Sundar is currently working as senior Professor in the Department of Biotechnology and Dean in the School of Bio and Chemical engineering in Kalasalingam Academy of Research and Education. His research interests in Immunology and Medical Vaccinology.

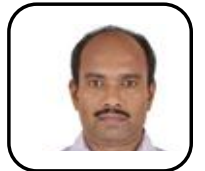

Dr. A. Muthukumaran is currently working as Associate Professor and Head in the Department of Biotechnology in Kalasalingam Academy of Research and Education. His research interests in Nanobiotechnology, Drug delivery and Safety

assessment of nanoparticles 